\title{
Diversity and toxic potential of algal bloom forming species from Takaroa lagoon (Tuamotu, French Polynesia): a field and mesocosm study
}

\author{
Martine Rodier*, Sébastien Longo, Kevin Henry, André Ung, Alain Lo-Yat, \\ H. Taiana Darius, Jérôme Viallon, Beatriz Beker, Bruno Delesalle, Mireille Chinain \\ *Corresponding author: martine.rodier@ird.fr \\ Aquatic Microbial Ecology 83: 15-34 (2019)
}

Table S1: List of bloom events and associated environmental impacts reported from French Polynesian lagoons by either local populations, public authorities or researchers.

\begin{tabular}{|c|c|c|c|}
\hline YEAR & ATOLL & BLOOM EVENT/IMPACTS & REFERENCES \\
\hline before 1906 & Hikueru & discolored waters & Hervé, 1934b \\
\hline 1907 & Taiarao & red waters & Hervé, 1934b \\
\hline 1920 & Hikueru & $? ? ?$ & Hervé, 1934b \\
\hline 1953 & Mataiva & green waters & Conte, 1989a \\
\hline Jan. 1964 & Tahiti & fish mortalities & La Dépêche de Tahiti 1994a \\
\hline Feb. \& July 1970 & Hikueru & copepods proliferation & Taxit \& Reed, 1971 ; Stein, 1971 \\
\hline Jan. \& Feb. 1970 & Tupai & ??? & Stein 1971 \\
\hline Feb. 1971 & Hikueru & zooplancton proliferation, brown waters & Taxit \& Reed, 1971 ; Stein, 1971 \\
\hline Nov. 72 \& Feb. 73 & Takume & mass mortalities & Galenon, 1980 \\
\hline 1974 & Marquises & Aulacodiscus kittonii var. africanus & Sournia \& Plessis, 1974 \\
\hline 1976 & Tupai & Polykrikos sp. & Landret, 1976a \\
\hline 1978 & Tahiti & fish mortalities & Galzin, 1979a \\
\hline 1978 & Marquesas & Gymnodinium + Amphidinium & Taxit 1978 \\
\hline $1974 \& 1979$ & Takume & brown waters & Galenon 1980 \\
\hline 1979 & Hikueru & $? ? ?$ & $\mathrm{DRMM}^{\mathrm{a}}$ \\
\hline 1980 & Hikueru & zooplankton proliferation & Galenon 1980 \\
\hline June 1980 & Takume & $? ? ?$ & Galenon 1980 \\
\hline 1983 & Tetiaroa & $? ? ?$ & DuPrela $^{a}$ \\
\hline July 1984 & Tupai & Polykrikos sp. & Delesalle, 1990 \\
\hline April 1994 & Hikueru & coccoides chlorophyceae & Harris \& Fichez, 1995 \\
\hline April 1994 & Manihi & Proboscia alata+ Dinoflagellés & Delesalle, 1994b \\
\hline April 1994 & Arutua & $? ? ?$ & $\mathrm{DRMM}^{\mathrm{a}}$ \\
\hline April. 1994 & Faaite & $? ? ?$ & $\mathrm{DRMM}^{\mathrm{a}}$ \\
\hline Dec. 1996 & Takapoto & chlorophyceae & Loreta $^{a}$ \\
\hline Feb.1997 & Manihi & coccolithophorides & Delesalle ${ }^{a}$ \\
\hline July. 1997 & Manihi & Proboscia alata & Delesalle ${ }^{a}$ \\
\hline Oct. 1997 & Amanu & Proboscia alata & Delesallea \\
\hline Feb. 1998 & Océan & Oscillatoria & Payri, Loreta \\
\hline Feb. 2000 & Ahe & Scripsiella & Delesallea \\
\hline
\end{tabular}




\begin{tabular}{|c|c|c|c|}
\hline Feb. 2001 & Takaroa & Proboscia alata & Delesallea \\
\hline 2009 & Tatakoto & giant-clam mass-mortalities & $\mathrm{DRMM}^{\mathrm{a}}$ \\
\hline March 2012 & Fangatau & $? ? ?$ & DRMMa $^{a}$ \\
\hline June 2012 & Ahe & $? ? ?$ & DRMMa $^{a}$ \\
\hline Jan. 2013 & Tatakoto & giant-clam mass-mortalities & DRMMa $^{a}$ \\
\hline Feb. 2013 & Takapoto & $? ? ?$ & DRMM $^{a}$ \\
\hline Nov.2013 -Sep. 2014 & Takaroa & green waters, pearl oyster mortalities & Fougerouse, $2014^{b}$ \\
\hline Feb. 2017 & Pukarua & green waters & DRMMa $^{a}$ \\
\hline
\end{tabular}

a Field observations only, no official report

b Internal report

\section{LITERATURE CITED}

Delesalle B (1990) Ecologie du phytoplancton des lagons de Polynésie française. Thèse de doctorat, Ecole Pratique des Hautes Etudes, Perpignan

Delesalle B (1994) Phytoplancton des atolls de Manihi, Faka rava, Rikitea, Marutea Sud et Nengo Nengo en avril 1994. Rapport ronéoté

Fougerouse A, Dal Frarra M, Haumani G, Lo C (2014) Efflorescence algale dans le lagon de Takaroa. Rapport de mission. DRMM

Galenon (1980) Rapport de mission: Phénomènes des eaux rouges à Takumé, Documentation EVAAM, No. 997/Pêche (10/07/80)

Harris P, Fichez RP (1995) Observations et mécanismes de la crise dystrophique de 1994 dans le lagon de l'atoll d'Hikueru (Archipel des Tuamotu, Polynésie française). Notes et Documents Orstom, No. 45

Hervé F (1934) Extrait du Bulletin de l’Agence Générale des Colonies, 297 et 298

Sournia A, Plessis YB (1974) A red-water diatom, Aulacodiscus Kittonii var. africanus, in Marquesas Islands, Pacific Ocean. Bot Mar 17: 124-124

Stein S (1971) Etude spéciale du lagon d'Hikueru et réflexions sur les causes de mortalité des nacres de ce lagon en 1971 Service des Pêches, $N^{\circ} 69 /$ Pêche (26/02/69)

Taxit R (1978) Les phénomènes d'eaux rouges aux îles Marquises. Cah Pacifique 21: 285-292

Taxit R, Reed W (1971) Eaux colorées à Copépodes, CEP/SMCB, No. 950 